\title{
Effects of Long Non-Coding RNA MALAT1 Induced by High Glucose on the Biological Characteristics in Stroke
}

\author{
L. WANG, J. Q. PENG AND G. XIE* \\ Department of Rehabilitation, Liyuan Hospital, Tongji Medical College, Huazhong University of Science and Technology, \\ Hubei 430077, China
}

Wang et al.: Effects of Long Non-Coding RNA MALAT1 on the Biological Characteristics in Stroke

To investigate the effect of high glucose-induced long non-coding ribonucleic acid metastasis associated lung adenocarcinoma transcript 1 on the biological properties of stroke cells. Rat brain micro vascular endothelial cells were cultured in vitro and divided into non-high glucose culture group and high glucose culture group. Long non-coding ribonucleic acid- metastasis associated lung adenocarcinoma transcript 1 was overexpressed and silenced by cell transfection, and the expression levels of long non-coding ribonucleic acid- metastasis associated lung adenocarcinoma transcript 1 and miR-181a-5p were detected by reverse transcription polymerase chain reaction under different culture methods. The Cell TiterBlue assay was also used to analyze the changes of cell viability after various transfection treatments; the double fluorophore reporter gene assay was used to verify the targeting effect of long non-coding ribonucleic acid- metastasis associated lung adenocarcinoma transcript 1 and miR-181a-5p. The results showed that non-high glucose cultured rat brain micro vascular endothelial cells significantly down-regulated the expression of Long non-coding ribonucleic acid- metastasis associated lung adenocarcinoma transcript 1 compared to high glucose cultured rat brain micro vascular endothelial cells, but the expression level of miR-181a-5p was significantly increased with statistically significant differences $(p<0.05)$. Compared with the control group, the viability of rat brain micro vascular endothelial cells expression long non-coding ribonucleic acid- metastasis associated lung adenocarcinoma transcript 1 was significantly enhanced $(\mathbf{p}<\mathbf{0 . 0 5})$, while silencing long non-coding ribonucleic acid- metastasis associated lung adenocarcinoma transcript 1 significantly inhibit the viability of rat brain micro vascular endothelial cells $(\mathbf{p}<0.05)$. Reverse transcription polymerase chain reaction experiments showed that long non-coding ribonucleic acidmetastasis associated lung adenocarcinoma transcript 1 regulated the viability of rat brain micro vascular endothelial cells with miR-181a-5p expression levels at a positive correlation level, while dual fluorophore enzyme reporter gene assays suggested a region of complementary binding between long non-coding ribonucleic acid- metastasis associated lung adenocarcinoma transcript 1 and miR-181a-5p. Long noncoding ribonucleic acid- metastasis associated lung adenocarcinoma transcript 1 can achieve the effect on the activity of rat brain micro vascular endothelial cells under high glucose stimulation by regulating miR-181a-5p.

Key words: Stroke, brain microvascular endothelial cells, high glucose, MALAT1, miR-181a-5p

Stroke is one of the leading causes of death and disability worldwide, accompanied by symptoms such as cerebral ischemia or hypoxia ${ }^{[1]}$. Ischemic stroke (IS) is mainly caused by cerebral artery thrombosis or embolic occlusion, which is also the main cause of long-term disability in patients ${ }^{[2]}$. Stroke has the characteristics of high morbidity, disability rate and mortality, and has a serious impact on patients' physical and mental health and life quality ${ }^{[3]}$. The injury and dysfunction of vascular endothelial cells play a key role in the initiation of IS. Current studies have shown that inflammation is closely related to IS, and inflammation is involved in the occurrence, development, plaque formation, plaque rupture, and almost the whole process of thrombosis ${ }^{[4,5]}$. Recently, abnormal expression of long non-coding RNA (lncRNA) has been found to be a common feature of many cancers. LncRNA metastasis associated lung adenocarcinoma transcript 1 (MALAT1) has been found to be overexpressed in a variety of cancers, such as in neuroblastoma, where MALAT1 is highly expressed and promotes cancer cell invasion and migration ${ }^{[6]}$. Meanwhile, MALAT1 can act as the endogenous competitive ribonucleic acid (RNA) of various microRNA, for example, in prostate cancer, MALAT1 
inhibits cell apoptosis and promotes its proliferation by down-regulating MiRNA-1 ${ }^{[3]}$. At present, the mechanism between MALAT1 and miRNA in IS is not clear. Recent studies have shown that miR-181a-5p not only related to age, gastric cancer, colorectal cancer and esophageal squamous cell carcinoma ${ }^{[7]}$, but also plays an important role in the development of coronary heart disease $^{[8]}$. miR-181a-5p may be regulated by lncRNA MALAT1, while miR-181a-5p has been shown to be an important molecule regulating vascular endothelial cell activity and proliferation ${ }^{[9]}$. Therefore, this study hypothesized that lncRNA MALAT1 could participate in the abnormal activity of vascular endothelial cells caused by high glucose by regulating miR-181a-5pS, and designed in vitro experiments for verification.

Rat brain microvascular endothelial cells (rBMECs) were isolated, cultured and provided from rat brain by Guangzhou Lederenkang Biotechnology Co., LTD. DMEM medium, fetal bovine serum, Lipofectamine 2000 kit, Lipofectamine RNAiMAX, TRIzol reagent and TaqManTM Advanced miRNA cDNA synthesis kit were all purchased from Thermo Scientific (China) Co., Ltd. pcDNA3. 1-MALAT1, MALAT1-siRNA, miR181a-5p mimic, miR-181a-5p inhibitor and irrelevant sequences were purchased from Guangzhou Ruibo biology. Celltiter-blue kit and dual-luciferase reporter plasmid and dual-luciferase reporter gene detection system were purchased from Shanghai Promege Biological Products Co., Ltd. Primers for miR-181a-5p and U6 were purchased from QIAGEN.

Rat brain microvascular endothelial cells (rBMECs) were placed in DMEM medium containing $20 \%$ fetal bovine serum and placed vertically for $5 \mathrm{~h}$ in an incubator at $37^{\circ}$ and $5 \% \mathrm{CO}_{2}$. After cell adhesion, the cell culture flask was placed horizontally for further culture. The solution was changed once every $3 \mathrm{~d}$. When the cells grow to about $80 \%$ of the culture flask, they are routinely passaged. The cells after 2 generations were selected for subsequent experimental operation. Nonhyperglycemia treated cells were routinely cultured (medium glucose concentration was $5 \mathrm{mmol} / \mathrm{l}$ ), and cells requiring hyperglycemia were cultured in medium with glucose concentration of $25 \mathrm{mmol} / 1$.

Eukaryotic origin plasmid PCdNA3.1-MALAT1 and MALAT1-siRNA were transplanted to rBMECs to achieve overexpression and silence respectively in cell. The overexpression and silencing of miR-181a-5p in the cells were achieved by transfecting rBMECs with miR-181a-5p analogies and miR-181a-5p inhibitor. The plasmid was transfected with Lipofectamine 2000 reagent, and the pcDNA3.1 empty plasmid was used as a transfection control. Lipo-fectamine RNAiMAX reagent is used for transfection of MALAT1-siRNA, miRNA mimic and inhibitor sequences. Use cells transfected with irrelevant sequences as transfection controls.

The cells were divided into non-high-glucose culture group and high-glucose culture group according to the difference of high-glucose stimulation and transfection. The non-high-glucose culture group was further divided into 3 subgroups, including the negative transfection control subgroup, the PCDNA3.1-MALAT1 transfection subgroup, and the co-transfection subgroup of PCDNA3.1-MALAT1+miR-181a-5p mimic. The high glucose culture group was also divided into 3 subgroups, including the negative transfection control subgroup, the transfer MALAT1-siRNA subgroup, and the MALAT1-siRNA+miR-181a-5p inhibitor cotransfection subgroup.

Total RNA was extracted from cell samples in each group using TRIzol reagent. The reverse-transcription of miRNA was performed using the Prime-Scripttm RT kit. After reverse transcription was completed, cDNA was quantitatively amplified by SYBR Premix Ex TaqTM kit, with GAPDH as the internal reference. The TaQ-ManTM Advanced miRNA cDNA synthesis kit was used for reverse transcription of MicroRNA, and miScript SYBR Green PCR kit was used for quantitative fluorescence amplification of cDNA after reverse transcription, and U6 was used as the standard.

After obtaining the cycle threshold $(\mathrm{Ct})$, use the 2- $\Delta \Delta \mathrm{Ct}$ method to calculate the relative level of the target gene. The upstream primer sequence of IncRNA-MALAT1 is CCTCGAGACAAAGAGCCCTCTGCACTAG-3', the downstream primer sequence is 5' - A T C G G A T C C G A G C A A A T G GAGACAAAGGAC-3'; the upstream primer sequence of GAPDH is 5 ' - C AT G T T C G T C AT G G G G T G A A C C A - 3', and the downstream primer sequence is 5 '-AGTGATGGCATGGACTGTGGTCAT-3'. miR181a-5p and U6 both adopt commercially available miscript primers.

Cells in each group were planted in 96-well plates at A density of 5,000 cells per well. The absorbance (A) value at $573 \mathrm{~nm}$ wavelength of cells in each group was determined $24 \mathrm{~h}$ and $72 \mathrm{~h}$ after implantation using celltiter-Blue kit. The relative changes of cell activity between $72 \mathrm{~h}$ and $24 \mathrm{~h}$ were calculated according to the 
formula. Relative activity of cells $(\%)=72 \mathrm{~h} \mathrm{~A}$ value $/ 24$ h A value $\times 100 \%$.

Double luciferase reporter gene assay was used to confirm the binding ability of IncRNA-MALAT1 to miR-181a-5p: a wild-type lncRNA-MALAT1 luciferase reporter gene plasmid was constructed without any mutation and named as pmirGro-MALAT1-WT. At the same time, the binding region predicted by the above bioinformatics in IncRNA-MALAT1 was mutated to construct a mutant lncRNA-MALAT1 luciferase reporter gene plasmid, named pmirGro-MALAT1MUT. Use miR-181a-5p mimic (while using irrelevant sequences as negative control) and the above two luciferase reporter gene plasmids to co-transfect rBMECs, and then use the dual luciferase reporter gene detection system to determine the relative luciferase activity of the transfected cells.

SPSS 20.0 software was used for analysis. Quantitative data were expressed as $\mathrm{X} \pm \mathrm{S}$, homogeneity of variance analysis and $\mathrm{T}$ test were used for data analysis, and $\mathrm{p}<0.05, \mathrm{p}<0.01$ were considered statistically significant.

The results showed that the relative level of lncRNAMALAT1 in the high-glucose culture group was significantly higher than that of the non-high-glucose culture group $(\mathrm{P}<0.01)$ (fig. $1 \mathrm{~A})$, while the relative level of miR-181a-5p in the high-glucose culture group was significantly less than that of the non-high-glucose culture group $(p<0.01)$ (fig. 1B). This result suggested that high glucose stimulation can increase the level of lncRNA-MALAT1, but inhibit the expression of miR181a-5p.

For non-high-glucose rBMECs, the relative activity of cells was significantly increased after transfected with PCDNA3.1-MALAT1 for $72 \mathrm{~h}$ compared with the negative transfection control subgroup $(p<0.01)$.
For rBMECs cultured with high glucose, the relative viability of cells transfected with MALAT1-siRNA for $72 \mathrm{~h}$ was significantly reduced compared with the negative transfection control subgroup $(\mathrm{p}<0.01)$. The relative cell viability of the negative transfection control subgroup in the high-glucose culture group was significantly higher than that of the negative transfection control subgroup in the non-high-glucose culture group $(\mathrm{p}<0.01)$. More importantly, in the nonhigh glucose culture group, the cell viability of the cotransfected subgroup of pcDNA3.1-MALAT1+miR181a-5p mimic was significantly lower than that of the pcDNA3.1-MALAT1 transfected subgroup $(\mathrm{p}<0.05)$, but higher than that of the negative transfection control subgroup $(\mathrm{p}<0.05)$. In the high glucose culture group, the relative viability of the cells in the MALAT1siRNA+miR-145 inhibitor co-transfection subgroup was significantly higher than that in the MALAT1siRNA subgroup $(\mathrm{p}<0.05)$, but lower than that of the negative transfection control subgroup $(\mathrm{P}<0.05)$, as shown in fig. 2. The above results suggested that high glucose treatment can induce the activity of rBMECs, IncRNA-MALAT1 can promote the activity of rBMECs, and miR-181a-5p can inhibit the activity of rBMECs. The regulation of lncRNAMALAT1 on rBMECs activity depends on the levels of miR-181a-5p.

The RT-qPCR results showed that the relative levels of miR-181a-5p transfected with pcDNA3.1-MALAT1 subgroup were significantly reduced compared with the negative transfection control subgroup $(\mathrm{p}<0.05)$, and the relative level of miR-181a-5p transfected with MALAT1-siRNA subgroup was significantly higher than that of the negative transfection control subgroup $(\mathrm{p}<0.05)$, as shown in fig. 3. These results suggested that IncRNA-MALAT1 can inhibit the expression of
A

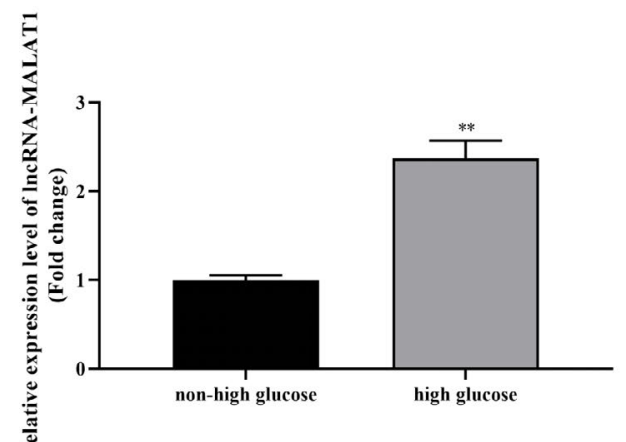

B

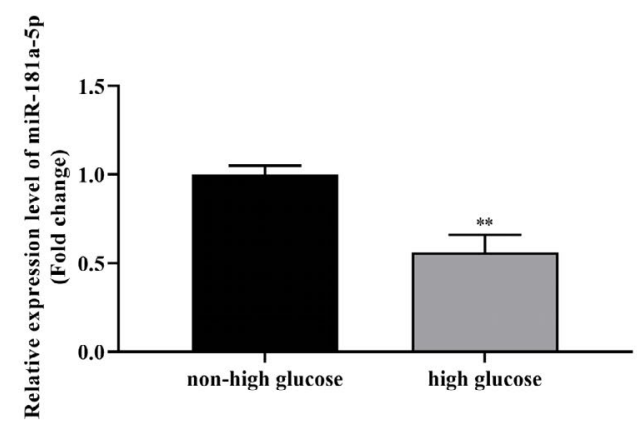

Fig. 1: The levels of IncRNA-MALAT1 and miR-181a-5p in two groups $(\square)$ : Non high glucose, $(\square)$ : High glucose
Changes in the levels of IncRNA-MALAT1 in vascular endothelial cells cultured in non-high-glucose and high-glucose; (B) Changes in the levels of miR-181a-5p in vascular endothelial cells cultured in non-high-glucose and high-glucose; $* * p<0.01$, compared with non-high-glucose

22

Indian Journal of Pharmaceutical Sciences

Special Issue 3, 2021 
miR-181a-5p. The results of the dual luciferase reporter gene experiment showed that compared with cells cotransfected with miR-181a-5p mimic and pmirGLOMALAT1-MUT plasmids, the relative luciferase activity of the cells co-transfected with miR-181a-5p mimic and pmirGLO-MALAT1-WT was significantly reduced $(\mathrm{p}<0.05)$, as shown in fig. 4 . These results suggested that IncRNA-MALAT1 can indeed bind directly to miR-181a-5p, which may inhibit the expression of miR-181a-5p in the form of "nucleic acid sponge".

Stroke is the third leading cause of death in humans, which brings a heavy social and economic burden to patients and society. Its pathophysiological mechanisms are very complex, including nerve regeneration, inhibition of cell apoptosis, neuroinflammatory response and angiogenesis ${ }^{[10]}$. Stroke involved in the multi-layer cascade mediation of molecular mechanisms, partly through post-transcriptional activity regulation ${ }^{[11]}$. Therefore, the study of post-transcriptional regulation of stroke genes has important clinical significance.

Cerebral atherosclerosis is a major cause of stroke, and the death in more than $80 \%$ stroke patients is related

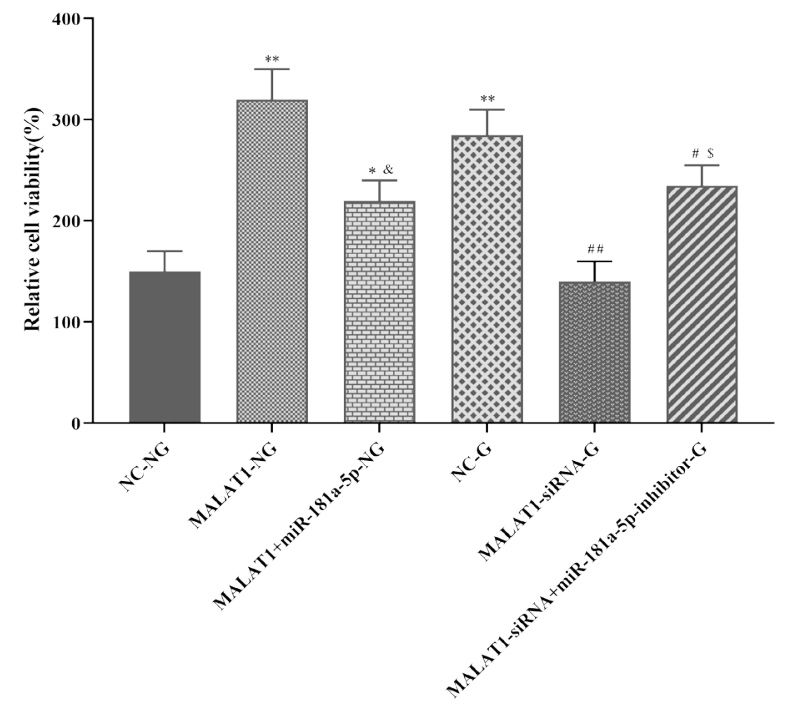

Fig. 2: Changes cell viability of rBMECs after different transfection in two groups

NC-NG: Non high glucose in negative transfection control subgroup; MALAT1-NG: Non-high glucose in pcDNA3.1MALAT1 transfected subgroup; MALAT1+miR-181a-5pNG: Non-high glucose in pcDNA3.1-MALAT1+miR-181a-5p mimic transfected subgroup; NC-G: High-glucose in negative transfection control subgroup; MALAT1-siRNA-G: Highglucose in MALAT1-siRNA transfected subgroup; MALAT1siRNA+miR-181a-5p inhibitor G: High-glucose in MALAT1siRNA+miR-181a-5p inhibitor transfected subgroup, * $p<0.05$, compared with NCNG; ${ }^{\#} \mathbf{p}<0.05$, compared with NC-G; ${ }^{\&} \mathbf{p}<0.05$, compared with MALAT1-NG; ${ }^{\mathrm{s}} \mathbf{p}<0.05$, compared with MALAT1-siRNA-G; **p<0.01, compared with NC-NG; ${ }^{\#} \mathbf{p}<\mathbf{0 . 0 1}$, compared with NC-G.

Special Issue 3, 2021

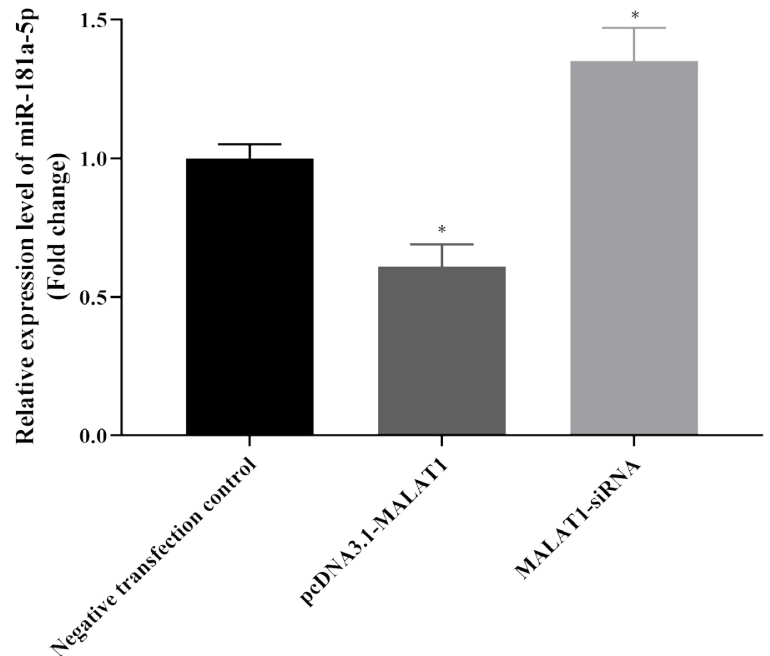

Fig. 3: miR-181a-5p levels effected by IncRNA-MALAT1 expression in rBMECs

$* \mathbf{p}<0.05$, compared with the negative transfection control subgroup

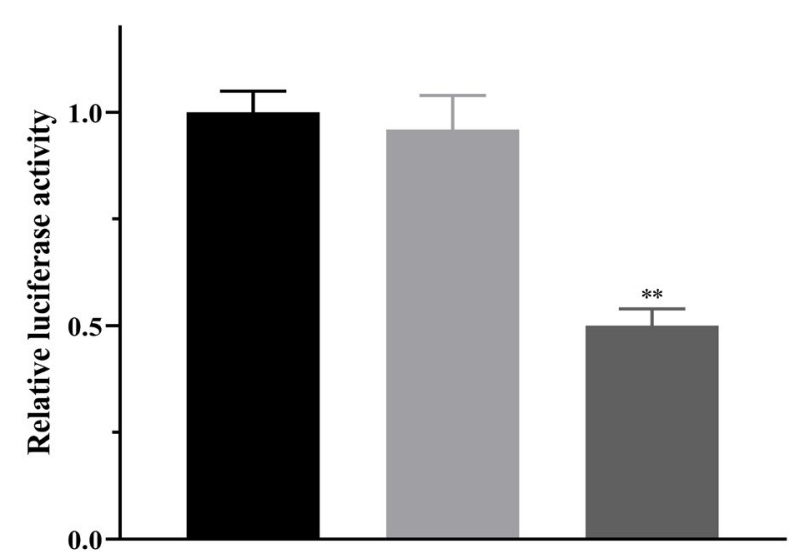

Fig. 4: Changes in relative luciferase activity of cells in double luciferase reporter gene assay

${ }^{*} \mathbf{p}<0.01$, compared with the cells co-transfected with miR-181a5p mimic and pmirGLO-MALAT1-MUT subgroup

to cardiovascular and cerebrovascular events caused by atherosclerosis ${ }^{[12,13]}$. Stroke patients often accompanied by hyperglycemia, hyperinsulinemia, and lipoprotein metabolism disorders. High insulin levels can stimulate the growth of endothelial cells and smooth muscle cells, while hyperglycemia and insulin resistance can damage endothelial cells. These effects are considered to be an important pathophysiological link in the formation of atherosclerosis in stroke patients ${ }^{[14,15]}$. Therefore, to prevent the excessive proliferation of rBMECs with certain interventions may be of great significance to the prevention and treatment of stroke vascular diseases. Hyperglycemia is one of the main characteristics of stroke, and it has been proven to be an independent risk factor for atherosclerosis in stroke patients ${ }^{[10,12]}$. Therefore, it has great clinical significance to explore the mechanism of hyperproliferation in rBMECs caused by high glucose and design intervention 
measures. ncRNA is a type of non-coding RNA with a transcript of more than 200 nucleotide units. Although it does not code for protein, it plays an important role in chromatin remodeling, transcription regulation, posttranscriptional regulation and protein metabolism. This study focuses on the role of IncRNA-MALAT1 in the rBMECs hyperproliferation caused by hyperglycemia. IncRNA-MALAT1 has been confirmed to be involved in vascular diabetes associated disease complicated. Studies have found that there is a high expression of lncRNA-MALAT1 in the retinal microvascular tissue of a diabetic rat model, but no studies confirmed that there is also a high expression of lncRNA-MALAT1 in vascular endothelial cells cultured in high glucose and it can promote vascular endothelium cell dysfunction and whether it is related to the excessive proliferation of rBMECs under high glucose. In this study, we found that rat $\mathrm{rBMECs}$ stimulated by high glucose showed high expression of lncRNA-MALAT1 via cell experiments in vitro, while the increased cell activity induced by high glucose stimulation was weakened after the expression of IncRNA-MALAT1 was inhibited. After up regulating the IncRNA-MALAT1 of rBMECs in non-high-glucose culture condition, we found that the cell activity increased. Therefore, this study considered that IncRNA-MALAT1 could promote the activity of rBMECs and participate in the abnormal activity of rBMECs caused by high glucose.

The regulatory mechanism of lncRNA is quite complicated. One of the most important mechanisms is competitive endogenous RNA theory, that is, lncRNAs can act as "nucleic acid sponges", to adsorb corresponding complementary microRNAs, resulting in the reduction of free molecules of the microRNA, which indirectly leads to the change of target gene level of this microRNA ${ }^{[18]}$. Based on this theory, this study considered that IncRNA-MALAT1 may work through certain microRNA. Studies have confirmed that miR$181 \mathrm{a}-5 \mathrm{p}$ is the most abundant microRNA in rBMECs, which can not only regulate the phenotype of rBMECs but also regulate their proliferation and migration. Studies have pointed out ${ }^{[19]}$ that hyperglycemia can inhibit the expression of miR-181a-5p in rBMECs, and miR-181a-5p itself has the effect of inhibiting the proliferation of rBMECs. Therefore, miR-181a$5 \mathrm{p}$ is likely to participate in vascular disease caused by diabete, especially it may be involved in the damage of rBMECs in this process ${ }^{[20]}$. On the basis of bioinformatics analysis, this study modulated the lncRNA-MALAT1 level of rBMECs by transfection and detected the expression changes of miR-181a-
$5 \mathrm{p}$. The results suggested that IncRNA-MALAT1 can inhibit the miR-181a-5p level. Further dual-luciferase reporter gene experiments showed that lncRNAMALAT1 could indeed combine with miR-181a-5p in a complementary manner. In addition, this study also found that miR-181a-5p had a low expression in rBMECs stimulated by high glucose, and indeed played a role in inhibiting the activity of rBMECs, which was consistent with previous studies.

Based on these results, we believe that IncRNAMALAT1 can affect the activity of rBMECs under high glucose stimulation by regulating miR-181a-5p.

\section{Acknowledgements:}

This work was supported by Liyuan Hospital, Tongji Medical College, Huazhong University of Science and Technology.

\section{Conflicts of interest:}

The authors declared no conflict of interest.

\section{REFERENCES}

1. Hu X, De Silva TM, Chen J, Faraci FM. Cerebral vascular disease and neurovascular injury in ischemic stroke. Circ Res 2017;120(3):449-71.

2. Urdaneta AE, Bhalla P. Cutting edge acute ischemic stroke management. Emerg Med Clin North Am 2019;37(3):365-79.

3. Jarroux J, Morillon A, Pinskaya M. History, discovery and classification of lncRNAs. Adv Exp Med Biol 2017;1008:1-46

4. Ji P, Diederichs S, Wang W. MALAT-1, a novel noncoding RNA, and thymosin beta4 predict metastasis and survival in early-stage non-small cell lung cancer. Oncogene 2003;22(39): 8031-41.

5. Gutschner T, Hämmerle M, Eißmann M, Hsu J, Kim Y, Hung $\mathrm{G}$, et al. The noncoding RNA MALAT1 is a critical regulator of the metastasis phenotype of lung cancer cells. Cancer Res 2013;73(3):1180-9.

6. Jiao F, Hu H, Han T, Yuan C, Wang L, Jin Z, et al. Long noncoding RNA MALAT-1 enhances stem cell-like phenotypes in pancreatic cancer cells. Int J Mol Sci 2015;16(4):6677-93.

7. Sainson RC, Johnston DA, Chu HC, Holderfield MT, Nakatsu $\mathrm{MN}$, Crampton SP, et al. TNF primes endothelial cells for angiogenic sprouting by inducing a tip cell phenotype. Blood 2008;111(10):4997-5007.

8. Welser JV, Li L, Milner R. Microglial activation state exerts a biphasic influence on brain endothelial cell proliferation by regulating the balance of TNF and TGF- $\beta 1$. J Neuroinflammation 2010;7(1):1-7.

9. Yukami T, Yagita Y, Sugiyama Y, Oyama N, Watanabe A, Sasaki T, et al. Chronic elevation of tumor necrosis factor- $\alpha$ mediates the impairment of leptomeningeal arteriogenesis in $\mathrm{db} / \mathrm{db}$ mice. Stroke 2015;46(6):1657-63.

10. Zhang $X$, Tang $X$, Liu K, Hamblin MH, Yin KJ. Long noncoding RNA Malat1 regulates cerebrovascular pathologies in ischemic stroke. J Neurosci 2017;37(7):1797-806.

11. Yang Z, Cai X, Xu A, Xu F, Liang Q. Bone marrow stromal cell transplantation through tail vein injection promotes 
angiogenesis and vascular endothelial growth factor expression in cerebral infarct area in rats. Cytotherapy 2015;17(9):120012.

12. Xu X, Ge S, Jia R, Zhou Y, Song X, Zhang H, et al. Hypoxiainduced miR-181b enhances angiogenesis of retinoblastoma cells by targeting PDCD10 and GATA6. Oncol Rep 2015;33(6):2789-96.

13. Tee AE, Liu B, Song R, Li J, Pasquier E, Cheung BB, et al. The long noncoding RNA MALAT1 promotes tumor-driven angiogenesis by up-regulating pro-angiogenic gene expression. Oncotarget 2016;7(8):8663- 75.

14. Li X, Song Y, Liu F, Liu D, Miao H, Ren J, et al. Long noncoding RNA MALAT1 promotes proliferation, angiogenesis, and immunosuppressive properties of mesenchymal stem cells by inducing VEGF and IDO. J Cell Biochem 2017;118(9):278091.

15. Michalik KM, You X, Manavski Y, Doddaballapur A, Zörnig $\mathrm{M}$, Braun T, et al. Long noncoding RNA MALAT1 regulates endothelial cell function and vessel growth. Circ Res 2014;114(9):1389-97.

16. Brewer JW, Hendershot LM. Building an antibody factory: a job for the unfolded protein response. Nat Immunol 2005;6(1):23-9.

17. Yuan Y, Guo Q, Ye Z, Pingping X, Wang N, Song Z. Ischemic postconditioning protects brain from ischemia/reperfusion injury by attenuating endoplasmic reticulum stress-induced apoptosis through PI3K-Akt pathway. Brain Res 2011;1367:8593.

18. Choi AY, Choi JH, Yoon H, Hwang KY, Noh MH, Choe W, et al. Luteolin induces apoptosis through endoplasmic reticulum stress and mitochondrial dysfunction in Neuro-2a mouse neuroblastoma cells. Eur J Pharmacol 2011;668(1):115-26.

19. Ferrara N. Vascular endothelial growth factor: basic science and clinical progress. Endocr Rev 2004;25(4):581-611.

20. Schachtner H, Calaminus SD, Thomas SG, Machesky LM. Podosomes in adhesion, migration, mechanosensing and matrix remodeling. Cytoskeleton 2013;70(10):572-89.

This is an open access article distributed under the terms of the Creative Commons Attribution-NonCommercial-ShareAlike 3.0 License, which allows others to remix, tweak, and build upon the work non-commercially, as long as the author is credited and the new creations are licensed under the identical terms

This article was originally published in a special issue, "Evolutionary Strategies in Biomedical Research and Pharmaceutical Sciences" Indian J Pharm Sci 2020:83(3)

Spl issue;20-25 